\title{
Tratamiento Epistemológico de la Aporía Dicotómica.
}

Entre todas las "curiosidades" filosóficas de verdadero interés para el investigador, se destacan con llamativo perfil las aporías de Zenón.

Los cuatro argumentos en contra del movimiento, con los cuales Zenón dejó perplejos a los pensadores de su época, se han mantenido incólumes a través de la Historia. Las aporías eleatas, han agitado el pensamiento de los filósofos, y han promovido mil disputas interminables.

Sin embargo, a pesar de las múltiples soluciones, ninguna ha logrado"derfuir las bases de la argumentación zenoniana. ¿A qué causa se debe este extraño fenómeno? ¿Porqué los ensayos de los más pođerosos teorizadores se han estrellado contra la muralla de los eleatas, más inconmovible que el mismo ser por ellos invocado?.

El presente ensayo trata de dar una respuesta satisfactoria a esta pregunta. Para ello compara las principales soluciones, y las relaciona a la problemática planteada por el discípulo de Parménides. De esta manera pretende demostrar, que la multiplicidad-muchas veces contradictoria-de las soluciones erradas, se debe a una dirección resolutoria equivoca. El problema ha sido encarado metafí- 


\section{$-338-$}

sicamente. Y sólo se puede llegar a una visión certera de él, desde un punto de vista epistemológico...

De las cuatro famosas aporías, sólo tomaremos la primera, es decir, la aporía de la Dicotomía, por ser el factor común a la que se reducen las restantes.

"Una flecha no podrá nunca alcanzar su blanco, porque cuando haya recorrido la mitad del camino, le faltará todavía la mitad, y cuando haya recorrido la mitad de esta mitad, le faltará la mitad de la mitad, y así "in infinitum". (Este fragmento no se encuentra entre los poquísimos originales de Zenón. Es Aristóteles quien refiere el argumento por vez primera. Física, libro Z).

La sencillez misma del argumento, demuestra su fuerza. A primera vista parece trivial, y se pudiera creer que es un mero juego de palabras.

Sin embargo, apenas el pensamiento penetra con mayor agudeza en la plenitud de su sentido, se percibe de inmediato su insondable profundidad. La aporía dicotómica, es una prueba de la capacidad de los pensadores helenos, los cuales en medid de sureleganteetverbalismo, tuvieron siempre las más hondas deiprimordiales jutelecciones filosóficas.

La exposición de las soluciones dadas a través de la Historia, más que sistemáticamente organizada, está orientada a hacer resaltar los defectos de la orientación general que ha prevalecido, y a hacer notar las posibilidades de una nueva interpretación.

\section{SOLUCION DE ARISTOTELES.}

Para el estagirita, todo el error estriba en la confusión de la potencia con el acto. La potencia es divisible, pero el acto es unitario, y como tal se opone a toda separación 
posible. Ahora bien el movimiento es un acto, es por 10 tanto unitario, indivisible. Por esa razón es falso el razonamiento de Zenón, cuya visión carecía de los suficientes fundamentos filosóficos.

\section{CRITICA.}

La crítica aristotélica-que tiene el mérito de ser la única argumentación antigua digna de tomarse en cuenta-es sin embargo demasiado elemental. Debido el dogmatismo imperante en el pensaminto helénico, (inevitable desde luego, si se considera las condiciones de paternidad que lo caracterizan) Aristóteles no analiza los conceptos de los cuales hace uso. En efecto ¿ por qué la potencia es divisible y no el acto? Todo acto (menos el acto puro) ¿nó es a su vez potencia respecto de un eidos de mayor jerarquía?. Entonces todo ente debe tener algo de divisibllidad porqué la potencia, aunque sea en grado mínimo, se incrusta en sil seno.

Además, la concepción del movimiento como un acto, ¿nó se contrapone a su definiciôn más generalizada del movimiento, comerele pas6 de la potenciasal acto?.

\section{SOLUCION DE BERGSON.}

Según el pensador francés, la ilusión de los eleatas, se deriva, exclusivamente de la confusión entre el espacio y el movimiento.

Este punto de vista se deriva de la orientación general de su sistema, que se basa en el esfuerzo hacia una precisa delimitación entre lo homogéneo, coexistente y espacial, y lo heterogéneo sucesivo y temporal. El hombre, debido a exigencias de adaptación, dirige habitualmente sı 
conocimiento, hacia lo espacial, y elabora una serie de formas espaciales, que le sirven de instrumental cognoscitivo. Luego, a causa del hábito de conocer a través de estas formas, las aplica a regiones que no tienen nada que ver con lo espacial. $\mathrm{Y}$ a causa de esto surgen una serie de confusiones y de falsos problemas, que se disipan apenas se limita el campo de aplicabilidad de estas formas, y se aplica al campo de la temporalidad pura, formas cognoscitivas específicas.

Uno de los primeros ejemplos-de esta tal vez originariamente inevitable confusión-es el famoso sofisma de la Dicotomía.

Cuando Zenón afirma que la flecha jamás alcanzará el blanco, está sencillamente confundiendo el espacio, con el movimiento. Es decir está confundiendo lo homogeneo, aquello cuyas partes se excluyen y que es arbitrariamente divisible, con lo heterogéneo, con aquello que no se puede dividir en partes, porque es un todo unitario, cuyos sucesivos estados se hallan unitariamente compenetrados como las fases de una melodia.

El movimiento, se apoya en el espacio, pero no es el espacio mismo. No se da en el espacio. La percepción sólo nos presenta una serie Pdecestados difèrentes, sucesivos. Es nuestra sintesis mental, la que une estos estados, y la que, gracias al devenir sicológico, les presta la continuidad y la fluidez esenciales al movimiento. El movimiento es un proceso unitario indivisible (esto nos recuerda la posición aristotélica), y en cambio el espacio es un todo divisible al infinito, no unitario, compuesto por una "infinidad" de partes. (Nótese el giro metafísico de los conceptos bergsonianos).

De manera, pues que cuando Zenón, dice que la flecha jamás alcanzárá su blanco, lo único que está diciendo, es que el espacio recorrido por ella,- tiene una infinitud 
de partes, y nada más. Pero si se considera no el espacio, que es un mero soporte del movimiento, sino el movimiento mismo que no ocupa espacio, entonces se comprenderá perfectamente, porqué la flecha alcanza el blanco. La trayectoria de la flecha es indivisible en cuanto movimiento, y a cada estadía, corresponde una cantidad de espacio definido. Por esta razón, se entiende que rápidamente la flecha alcance, su objetivo, porque el espacio recorrido, no es la esencia del movimiento, sino es sólo la condición de s!1 desenvolvimiento unitario.

\section{CRITICA.}

La solución bergsoniana, es no cabe duda, la más aguda y original de todas. Sin embargo deja de lado, algunos hechos, que son dignos de consideración.

$\mathrm{El}$ argumento puede resumirse de la siguiente manera: se considera el movimiento, que es indivisible, luego se divide el espacio, que lo soporta, y a continuación, se quiere aplicar la división de este espacio al movimiento mismo.

En la solucióngbergsoniana empero,sce desbarata una aporía, pero se crea otra, tal vez más aguda. „En efecto, si el movimiento no ocupa espacio, no se puede dividir; pero la posición del móvil sí es espacial, y lo que se puede dividir es la distancia entre sus posiciones, no el movimiento con el cual las trascurre.

Si la argumentación bergsoniana es certera, se deriva apriori,

1) que el movimiento es inespacial,

2) que la distancia entre dos móviles es espacial.

Entonces cuando un móvil se acerca a otro, dos objetos espaciales, logran aproximarse mediante un proceso inespacial. 
¿Cómo es esto posible? ¿ Cómo explicarse que el espacio sea lo que sostiene o soporta (l'éspace sous-tend le mouvemente-Essai sur les donnés inmediates de la Conscience, De la multiplicité des états de Conscience, pag 86), al movimiento, es decir que sea la condición para que el movimiento exista, y que sin embargo el movimiento sea inespacial? (ibid pag. 87).

Si el movimiento se deriva de una sintesis mental, y lo que la percepción presenta en realidad, son fenómenos estáticos, ¿entonces el movimiento es un fenómeno subjetivo? La Sicología demuestra que hay percepción de movimiento, que el movimiento es una dación que no se elabora sintéticamente. Kant ya lo había visto muy bien cuando decía en la Crítica de la Razón pura, que el movimiento es un "modus empiricus" no determinable apriori.

De estas consideraciones se derivan dos consecuencias posibles:

I) o bien, la aporía zenoniana es falsa, pero entonces se cae en la aporía mucho más grave de la inespacialidad de lo espacial (porque, quej el movimientō seartemporal, no quiere decir que no sea también espacial) verso"

2) o bien la aporía de Zenón está bien planteada, en cuyo caso, hay que buscar otra solución.

\section{SOLUCION DE HAIMELIN.}

Para Hamelin, la realidad, es un proceso de composición categorial.

La categoría elemental es la relación. A ella se opone el número, y como síntesis de estas dos oposiciones nace el tiempo. Al tiempo que es exclusión irreversible, se opone el espacio que es la copresencia reversible. El movimiento es la síntesis del tiempo y del espacio. Es la posición en 
función de la duración y la duración en función de la posición.

Del movimiento, por el proceso dialéctico de la oposición y de la síntesis, se pasa a la cualidad, de esta a la alteración. La causalidad, la finalidad y la personalidad, forman la culminación del proceso categorial.

Si se acepta que la esencia del movimiento es la síntesis del espacio con el tiempo, se ve, según Hamelin, el error de Zenón. En efecto, en el planteamiento, de la aporía el filósofo griego, se olvida en absoluto del tiempo, tomando solo en cuenta al espacio. Su error no conciste en una confusión del espacio con el movimiento, sino en una visión unilateral de este último. Reduce el movimiento al espacio, y no se puede reducir lo más a lo menos.

Decir pues, que la flecha no alcanzará su destino, no tiene sentido, porque es presuponer que la flecha no se mueve o que efectúa un moviniento que no es movimiento. Si se toma en cuenta el movimiento como la síntesis del tiempo y del espacio, se comprenderá porqué el proyectil alcanza su meta, porquetelctienpo lentra aomo factor decisivo.

$$
\text { "Jorge Puccinelli Converso" }
$$

\section{CRITICA.}

Si en lugar de considerar solo el espacio recorrido, se considera además el tiempo, la aporía no solamente no pierde fuerza sino que se agudiza profundamente.

En efecto, supongamos que la flecha se halle a dos millas del lugar en que está el blanco, y que el tiempo que se demora una flecha para recorrer aquella distancia, sea de dos horas.

Cuando la flecha haya recorrido una milla-al cabo de una hora-le faltará todavía otra milla y otra hora. 
Cuando haya recorrido la mitad de lo que le falta, le faltará media milla y media hora. Cuando haya recorrido la mital de esta mitad, le faltará un cuarto de milla y un cuarto de hora (suponiendo desde luego que su velocidad sea uniforme, para poder efectuar la correlación directa entre el tiempo y el espacio).

Cuando haya recorrido la mitad de lo que le falta, le faltará todavía un octavo de milla y un octavo de hora. Y así "in infinitum". Por menos que le falte, tanto en espacio como en tiempo, siempre podrá dividirse esta cantidad infinitesimal, por la mitad. Y esta división puede prolongarse al infinito. Jamás llegará a un límite.

En esta aporía modificada, se ha tomado no sólo el espacio, sino tambien el tiempo. El movimiento está compuesto de espacio y tiempo, en cuanto dialécticamente unificados. Si la aporía solo valiese para el espacio, no se tendría derecho de afirmar del todo lo que solo vale de la parte.

En efecto, la ley de la relación genérico-específica, solo permite pasar del género a la especie, del todo a la parte, Pero lo que se dice del espacio, viale támbiẻn para el tiempo. $\mathrm{Y}$ como el movimiento no es sino la síntesis del espacio y del tiempo, lo que vale de ambos, vale de él, puesto que sus propiedades, no son sino la armonización de las propiedades de ambas categrorias inferiores, que separadamente se oponen, logrando unificarse en la estructura superior de movimiento.

\section{SOLUCION DE MESSER.}

La aporía de Zenón es una falsa aporía, puesto que resice $\epsilon_{n}$ un error capital: la confusión de lo discontinuo con lo continuo. 
-En efecto el espacio es discontinuo y el movimiento es continuo.

Lo que vale del espacio, no puede pues valer del movimiento, y vice versa.

\section{CRITICA.}

En el fondo la solución de Messer está basada en la de Bergson: la confusión del espacio con el movimiento.

Sólo que la debilidad de toda posición metafísica, se manifiesta en este caso con más fuerza, pues la afirmación metafísica es más punzante. En efecto ¿con que derecho se afirma que el espacio es discontinuo, y que el movimiento es continuo? ¿Por qué el espacio no va a ser continuo y ei movimiento discontinuo?

E1 espacio en sí, tal cual Messer trata de determinar, mediante una sola definición, no existe. Sólo se da un espacio biológico, un espacio físico, un espacio matemático, etc. Si Messer toma en cuenta al espacio biológico, su afirm?ción no tiene fündaniento pres biölógicamente el espacio es siempre continuog d se dresenta como una estructura resistencial, como una dificultad, para manejar las cosas. Una cosa está cerca, c1ando es fácil llegar a ella, y está lejos cuanco es difícil alcanzarla. Heidegger ha determinado con profunda intuición filosófica, el sentido biológicocomo el lo llama, "existencial"--del espacio. Y ha demostrado como el espacio no se presenta como un conjunto de partes, heterogéneas, sino como un impulso, como un esfuerzo de manuabilizar el mundo, de hacerlo nanejable.

Messer podría entonces acudir al espacio natemático. Pero aún en este caso se presentan serias di ficlitades. Matemáticamente, el espacio, no solo no es discontinuo, sino que por definición misma, es absolıtamente continuo. Es 
la manifestación más evidente de la continuidad. De manera pues, que la afirmación de que el espacio es discontinuo, es bastante infundada. Casi todos los filósofos han estado de acuerdo en que el espacio es continuo.

Otro tanto puede decirse del movimiento. ¿Es en verdad el movimiento tan continuo como parece? En algunos casos particulares, por lo menos, se ha demostrado lo contrario. El movimiento de irradiación energética, es quantico, es decir que carece de continuidad.

La solución de Messer, se basa en los errores cometidos por Zenón. Pero las afirmaciones que el hace ¿son ciertas?

Esto no quiere decir que Messer haya procedido sin conocimiento de causa. Seguramente cualquier otro filósofo hubiera cometido errores similares.

$\mathrm{Y}$ estos errores hacen pensar, en que la posición eleata, no es tan débil ni tan fácil de ser superada. En la aporía dicotómica se oculta un fondo de verdad, que se manifiesta en las posiciones forzadas, - tal vez más forzadas que la posición de Zenon liatqueadebe acudirse para poder ofrecer una solución, queeconro ise hả vistoendos casos anteriores, ofrecen un vacilante valor lógico.

\section{SOLUCION DE KOFFKA.}

El famoso representante de la Gestaltheorie, nos dice que Zenón comete un sofisma por "Petitio principii". "Es decir que para demostrar su tesis, "presupone" una tesis

En efecto que la flecha no pueda alcanzar el blanco, presupone que el tiempo no existe. Si solo hubiera espacio, la falacia de Zenón se trasformaría en verdad. Pero como hay un tiempo, y tiempo significa principio $y$ fin, el argumento de Zenón, carece de valor científico. 
Debido a que el hombre vulgar, por causas sicológicas, por exigencias de adaptación al medio, etc., está acostumbrado a separar el tiempo del espacio, es que se impresiona por el planteamiento eleata. Pero la realidad demuestra que no hay un tiempo sin un espacio.

\section{CRITICA.}

La posición de Koffka, está seguramente determinada por la Teoría de ia relatividad, la que en su tesis general, no cabe duda, tiene un elevado porcentaje de veracidad. Pero la adopción de la posición relativista, no esclarece el problema, sino que lo complica extraordinariamente.

Según el mismo Koffka, el movimiento es una traslación dentro del espacio, efectuada en un tiempo determinado; decir, la traslación dentro de algo que es infinito, en un lapso finito (determinado). Ahora bien ¿Cómo explicar que algo se puede dividir hasta el infinito, y su recorrido se efectúe en un tiempo fínito? ¿ Nó se está violando la ley fundamentalldelomovimiento?, atsaber: que los tiempos son proporcionales des espacios recorridos?.

¿Puéde conservarse la proporción, relacionando lo finito con lo infinito?.

\section{LA APORIA DE ZENON Y LA IRRACIONALIDAD INCLUIDA EN TODO SISTEMIA CIENTIFICO.}

Todos las antecedentes soluciones, tienen el mismo defecto: presuponer que la aporía zenoniana se base en un error de concepción. En otras palabras tratan de demostrar que la aporía no existe, que es una falsa aporía derivada de un sofisma por "Ignoratio elenchis".

Pero ¿ंes verdad esta suposición?. 
Las contradicciones inevitables en las que incurren los diversos filósofos que participan de esta opinión muestra a las claras, que la aporía de Zenón, no es tan fácil de resolver como pudiera parecer a primera vista...

$Y$ es que en el fondo-a pesar de los argumentos, muchas veces de profunda genialidad, de los filósofos tanto antiguos como modernos-, el argumento de Zenón está basado en una intuición cierta: la irracionalidad que presentan los principios fundamentales de las Ciencias.

El argumento general, que se repite en medio de sus múltiples formas es el siguiente: Zenón quiere reducir el movimiento al espacio. Si el movimiento fuera como el espacio, su aporía tendría valor. Pero como el movimiento es más qite el espacio, su antinomía no tiene valor.

No cabe duda que el movimiento es más que el espacio. Pero podemos prescindir del movimiento, quedándonos con el espacio, y sin embargo la aporía persiste.

Zenón podría decir: toda distancia es infinita, pues por más que se midal elcespacio entre un panto y otro, siempre quedará algoJpogemedixinelli Converso »

En efecto supongamos que un objeto esté a un metro de distancia de nosotros. Queremos medir exactamente esa distancia, y empezamos por medir la mitad de la distancia, la mitad de la mitad, etc. Dé esta manera la medición irá aumentando hasta el infinito.

Y lo mismo que se dice del espacio, se puede decir del tiempo. Zenón a este respecto podría plantear la siguiente aporía: la aporía del condenado a muerte inmortal. Supongamos que se ha condenado a muerte a un hombre, y que debe ser ejecutado a las doce del día en punto. Ahora bien, la hora de la ejecución nunca llegará, porque siempre po- 


\section{$-349-$}

drá dividirse el tiempo que falte-sea cual sea-por la mitad.

Entrando el tiempo y el espacio en la esencia del movimiento, podría decirse lo mismo de él.

Cualquiera argumentación que se haga en contra del argumento zenoniano, se estrella contra el aspecto de verdad que en el se vislumbra.

Solo que el aspecto de verdad que encierra el formidable argumento eleata, no es lo que su creador pretendía: la no existencia del movimiento.

La verdad suprahistórica que encierra el argumento de Zenón es la inevitable inadecuación entre la TEORIA y la PRACTICA, entre el conocimiento y lo conocido, entre el ontos y el nous. Zenón ha sido el primero de todos los pensadores en haber planteado-tal vez sin plena conciencia de lo que hacía-el problema de la irracionalidad del mundo.

Por eso la solución de la aporía dicotómica, no puede ser positiva, como han pretendido los filósofos que la han abordado, sino negativa. Todos han pretendido refutarla teóricamente, y demostrafinue Zenón había llegado al planteamiento de su aporía por falsos conceptos, o por ignorancia de los fenómenos. $Y$ han pretendido resolverla precisando el sentido filosófico de tiempo, del espacio y dal movimiento.

Esta es la causa profunda del fracaso de todos los contra argumentos. Por salirse de una aporía han caído en aporías mucho más difíciles, por apurar la salida del laberinto, han roto en su apresuramiento el hilo de Ariadna. $Y$ realmente que resolver una aporía, para caer en mayores cificultades, no vale la pena. Resolver un problema no consiste en cambiar las dificultades, sino en suprimirlas. 
Si se quiere reducir el argumento, a una mera validez espacial, como lo hace Bergson, se cae en mil dificultades, como hemos visto anteriormente, y además queda sin resolver la dificultad para el mismo espacio.

Bergson, extrae como consecuencia de su solución, que la Ciencia no se ocupa ni puede ocuparse del tiempo, sino que únicamente logra sistematizar relaciones espaciales.

Apenas la Ciencia quiere ingresar a un campo que no le corresponde, incurre en una serie de dificultades, cuyo máximo paradigma es la aporía de Zenón. La Ciencia solo llega a resultados certeros y perfectos, cuando se queda en su campo, a saber el campo de la espacial, de lo extenso, de lo que mutuamente se excluye. Pero si se anota que la aporía no solo se presenta en el estudio del tiempo y del movimiento, sino también en el estudio mismo del espacio, entonces la Ciencia no podría ocuparse del espacio. No se ocuparía por tanto de nada, y se negaría toda posibilidad de un conocimiento científico.

La Ciencia no solo estudia el espacio, sino también el tiempo. El cálculor por ejemplo, no se reduce a indicar las diversas posiciones que ocupa el móvil en el espacio, sino que nos indica el proceso del moverse en su más hondo dinamismo. Sólo que no puede indicarnos estos estadios de manera perfecta y acabada. $Y$ esto en general vale para todas las disciplinas científicas, en el más amplio sentido de la palabra.

La Ciencia estudia el espacio, el movimiento, el tiempo, la materia, la energía etc. peron no puede estudiar todos estos fenómenos de manera acabada, porque su instrumento de estudio no es acabado.

Esto es lo que no quiere reconocer Bergson, debido a 
su posición intuitivista general. "En su irracionalismo, llega a una posición racionalista. Por excluir a la Ciencia de ciertas regiones del Ser, le otorga un valor que la Ciencia no posee. La Ciencia tiene más y menos poder que el que le atribuye el famoso pensador francés. Más poder porque puede sistematizar casi todas las regiones del universo, inclusive las regiones de la más íntima temporalidad, y de la más absoluta compenetración. $\mathrm{Y}$ menos poder, porque a pesar de su amplio campo de aplicabilidad, nunca llega a sistematizaciones perfectas. Sus problemas y sus antimonias subsisten en el corazón de la espacialidad. En el estudio del espacio la Ciencia es tan imperfecta como en el estudio del movimiento o del tiempo puro. Puede ser que llegue a un mayor nivel de racionalidad, por ser las estructuras espaciales las más simples de todas, pero la racionalidad total, jamás pasará de ser una mera Idea en el sentido kantiano.

Otros, como Hamelin, dirán que el movimiento además del espacio consta del tiempo, etc. Pero se ve de inmediato que la poria eleata no solo vale para el espacio, sino también para el tienpop.

El sentido teórico general, del argumento del discípulo de Parménides, no es sino la expresión de la irracionalidad de la Ciencia a través del problema del "continuo". En la realidad se presenta como "dato" indiscutible el continuo. El espacio, el tiempo, el movimiento intuitivamente considerados son contínuos. Las formas son continuas. El continuo es lo que se contrapone a lo discreto, a lo separado y múltiple. Lo discreto es aquello cuyos elementos se excluyen. Así una pluralidad de objetos cualesquiera es algo discreto. Io mesas, 20 sillas, 30 hombres, son cantidades discretas. Cantidades contínuas son aquellas, cuyos elementos no se excluyen, 
sino que se puede pasar insensiblemente de los unos a los otros, como también lo ha establecido Rieman. Así las líneas, las formas, el espacio, el tiempo, el movimiento, son cantidades contínuas.

Pues bien, en la realidad se dan contínuos finitos. Un segmento de línea es un continuo, es decir una cantidad, que no tiene partes "separadas" unas de otras. $Y$ es un continuo finito. Lo mismo se puede decir del tiempo, del espacio etc.

La Ciencia puede racionalizar la cantidad discreta, de manera casi absoluta. En efecto, tratándose ce 50 hombres o 20 manzanas, la aporía de Zenón, no tiene sentido. Si se empieza a contar, desde el primer hombre, se llegará por cierto al trigésimo, sin mayores dificultades ni problemas.

Pero cuando se halla ante la cantidad continua, la Ciencia se encuentra con fuertes límites de su poder racionalizante.

En efecto, ta Ciencia no sólo quiere conocer la cantidad continua en sus aspectos y en sus formas, sino que también quiere "medirla. La racionalizacion no consiste solamente en la "determinación” perfecta, sino además en la aplicabilidad de la determinación. Un objeto en general, ha sido racionalizado, cuando se tiene dominio sobre él. Y solo se le puede dominar cuando se le ha "medido" (entiendo por medida la razón que indica la relación de continente a contenido o del todo a la parte), porque medir un objeto es poder predecir sus aspectos y sus posibilidades de presentación.

Cuando se mide un objeto que presenta relaciones esenciales (como son los objetos matemáticos contiviuas) conociendo la medida de uno de sus aspectos, se puede co- 
nocer la magnitud de los demás aspectos. Por eso el esfuerzo racionalizante del hombre tiende hacia la medida. Porque medir es prever, y prever es poder. Ya Einstein, con profundo sentido filosófico, ha dicho: en Física, lo que no se puede medir no existe, lo que equivale: en Física lo que no se puede medir no se puede racionalizar a fondo, no se puede dominar, ni aplicar a los fines de dominio que tiene el hombre frente al mundo.

Gracias a que se ha podido medir las formas cónicas, el tiempo y el espacio, es que se puede predecir los eclipses; gracias a que se ha podido medir la masa, la energía, etc., es que se puede predecir una serie de fenómnos naturales, conociendo simplemente algunos datos mínimos. Debido a este modo de racionalización es que ha surgido el portento de la Ciencia aplicada, que se basa casi exclusivamente en la medida del mundo natural.

Pero para medir la naturaleza, no solo hay que medir la cantidad discreta, fácil de medir. Hay que medir también la cantidad continua que es la base estructural de la Naturaleza. Prácticamento todoclo fundamental de la naturaleza es continuo. "Natura non datur saltus" decían los antiguos con profunda intuición.

Pero, aquí casualmente reside la dificultad. El único medio que posee el hombre para medir, es relacionar la parte con el todo, considerando al todo como la reunión de partes independientes. El todo es una suma de partes, he aquí el principio fundamental, en el que se ha basado la Razón durante miles de años y que ha traído tan enormes consecuencias en la cultura humana. Bien sea, porque el hombre lo primero que necesitó medir eran objetos diferentes unos de otros, que podían ser considerados como partes constitutivas de un todo (las diversas cabezas de ganado, 
los terrenos que constituían su patrimonio); bien sea por una limitación esencial a la Razón, lo cierto es que el hombre no posee otra manera de medir que la de relacionar el todo con una parte independiente, considerando al todo como la suma de una serie de partes idénticas que se excluyen mutuamente.

De esta modalidad de la medición en general es que se deriva la parcial irracionalidad de las Ciencias. ¡El hombre ha necesitado medir la cantidad continua, y la ha medido discretamente! Es decir que ha medido lo que no está compuesto de partes que se excluyen, como si en realidad lo estuviera.

Ha reducido las formas continuas, a la sucesión numérica. Los diferentes números indican las diferentes partes del continuo.

Pero el número, es comparado con otro número, una de las partes de un todo. Y estas partes se excluyen, porque los números se excluyen. Cada número es una de las partes del todo, y tiene su individualidad. E1 2 es completamente cistinto del 3 , y cada uno indica una parte distinta del todo que lo contiene. La parte puede ser equivalente, absolutamente reemplazable. Pero no por eso dejará de ser individualmente distinta.

Pues bien, la cantidad continua no presenta partes distintas unas de otras. Artificialmente se puede considerar de esta manera. Pero lógicamente hablando, la esencia de la continuidad es no saber donde empieza una parte ni donde termina. Todos los aspectos de la continuidad están perfectamente enlazados. No hay límite que separe a las partes del todo continuo. Entre dos aspectos de una recta solo se puede notar la diferencia de percepción. Pero jamás se po- 
drán separar con la absoluta e indiscutible precisión con que se separan el 2 y el 3 .

Como la única manera que tiene el hombre de medir, es evidenciar esta relación de la parte al todo debe-para medir lo continuo-considerarlo como formado de partes independientes. Pero como en realidad sus partes no son independientes, pronto se da cuenta que su medición es incompleta. Una recta por ejemplo, al ser medida, queda dividida en ro partes. Pero estas partes no constituyen partes independientes. Son meros puntos de referencia. De manera que el número IO, jamás podrá expresar la totalidad de las partes contenidas en el todo. Cada parte puede ser dividida en otras partes. Con el fin de precisar más esta medición inperfecta, el-entendimiento humano, efectúa el milagro cognoscitivo de los números fraccionarios, y llega al concepto sublime de infinito. Gracias a este prodigio de racionalización, gracias a la posibilidad de dividir el infinito, y consecuentemente de poder llegar casi a los últimos elementos de las formas continuas (los infinitésimos) se logra tuna mejor medición del continuo. Pero para esto hay que reconocer que un TODO FINITO, ESTA COMPUESTO DE UN INFINITO DE PARTES.

.Es decir hay que llegar a principios irracionales, en cuanto no están conformes con el principio supremo del entendimiento: el principio de no contradicción.

Esto y nada más que esto es lo que significa la famosa aporía de Zenón. Por eso desde el punto de vista teórico es perfectamente justificada.

Al decir que la flecha nunca alcanzará el blanco, Zenón nos dice sencillamente, que si un todo está compuesto 
de una infinidad de partes, es lógico que dicho todo sea infinito. Y nadie puede recorrer un todo infinito.

El equívoco de Zenón es epistemológico, no lógico, como han creído la mayor parte de sus críticos. Zenón no se equivoca ni sobre la naturaleza del movimiento, ni sobre el espacio, ni sobre el tiempo.

La falsedad de la aporía de Zenón no se deriva de creer que se puede aplicar al movimiento lo que se puede aplicar al espacio. No se deriva de partir de conceptos no demostrados. Se deriva de confundir la teoría con la práctica, la idea con la realidad. Teóricamente el espacio, el tiempo, el movimiento, las formas geométricas, y en general todo lo que es continuo, tiene una infinidad de partes. Porque solo así, se puede llegar a una parte que ya no se pueda dividir, y que en realidad se diferencie individualmente de las demás. (Condición indispensable de la mensurabilidad). Solo así se puede llegar a un último elemento de comparación mensurativa.

Pero en la realidad sucede todo lo contrario. La cantidad continua no esta constituída de una serie infinita de partes distintas. No hay verdadera distinción entre una parte y otra.

Esto ha traído como consecuencia la plena conciencia, de la imposibilidad de una racionalización perfecta de las cantidades continuas. La racionalización es solo aproximada. Jamás se podrá medir exactamente un continuo. Este es el profundo significado de la famosa "Teoría de los límites" en Análisis matemático. Y por esta misma razón, la "derivada" el instrumento más poderoso que ha inventado el hombre para medir la continuidad, se considera como un límite. 


\section{$-357-$}

No es otra tampoco la significación de las Geometrías puras. Las Geometrías puras tratan de excluir todo elemento métrico, de la consideración geométrica. De esta manera se consigue una racionalidad sin mácula. En efecto, no cabe duda que en la Geometría Descriptiva y en la Proyectiva la irracionalidad se reduce a un mínimo. La exactitud es perfecta. Pero no se puede medir. $Y$ por lo tanto, el servicio que prestan a la Ciencia aplicada es mucho menor que el que presta la Geometría analítica, y la Geometría corriente, que se basan en la medición numérica.

Zenón, debido al racionalismo obligado de su cultura, y a la confusión que siempre cometieron los helenos entre el ontos y el nous, el ser y el conocer, pasa de la contradicción cognoscitiva a la contradieción ontológica.

$Y$ este paso no tiene fundamento. Las categorías del ser no coinciden con las del conocer. En esta actitud histórica Zenón puede ser superado y rebatido. Pero en el planteamiento intrínsecó de la contradicción flagrante en que incurre el pensamiento, cuando quiere racionalizar to continuo, la posición de Zenón es eterna. Zenón se yergue como un coloso "en Ta Historia de la Filosofía.

Debido a su increíble penetración discursiva, y a su agudísima intuición, Zenón tiene derecho a pretender el título del "primer irracionalista de la Historia".

Francisco Mtró Quesada. 